\title{
Noise Reduction in Video Sequences - The State of Art and the Technique for Motion Detection
}

\author{
Reeja S R \\ Research scholar, RNSIT, Bangalore, VT \\ Asst.Professor, NMAMIT, nitte
}

\author{
N. P. Kavya, PhD. \\ HOD of MCA \\ RNSIT, Bangalore
}

\begin{abstract}
This paper provides a detailed state of the art of different video denoising techniques. Most of the video denoising algorithms are done through the motion detection technique. The main goal is to give a survey of various noise reduction techniques for video. Object detection is the first level of video denoising. The first level can be achieved through Motion Detection. This paper explained about the motion estimation and compensation techniques. The different video denoising techniques, motion detection techniques and the noises used are shown through the taxonomy.
\end{abstract}

\section{General Terms}

Image processing, motion detection

\section{Keywords}

Object tracking, frame differencing, image and video denoising.

\section{INTRODUCTION}

The process of noise removal noise from the video is called Video Denoising, where noise reduction in image can be done through the frame individually and between the frames. Video sequence noise reduction is used widely in traffic management, medical imaging and TV broadcasting applications. Noise reduction is an image restoration mechanism in which it attempts to recover image from a degraded image. Various denoising techniques make various assumptions, depending on the image as well as type of image. The paper [1] talked about motion detection in Real time video streams using moved frame background. Here the author considered first frame as background frame and comparing this frame with the current frame to get the difference. Moving object detection is the first step of video denoising as well as object tracking. This technique uses segmentation of moving objects from stationary background objects [2].

This is focused on higher level processing and decreases computation time. Shadow object segmentation is difficult and significant due to illumination changes. Most of the video denoising technique depends up on a single noise such as Gaussian or white noise. Different statistical distributions have been found in [35] with five major sources of noise. They are dark current noise and quantization noise. Whenever the real noisy images with noises from multiple sources are applied the performance of these denoising techniques will be decreased.

This paper organizes as follows. In the next section we recall the different techniques for video denoising, section 3 describes the various motion estimation and compensation and 4 describes the conclusion.

\section{VIDEO DENOISING -RELATED WORKS}

A wavelet domain spatial filter whose coefficients are manipulated using a Markov Random Field (MRF) image model has been proposed in [1]. In the Literature survey of [16] the authors proposed the Spatial (2-Dimentional) and spatio-temporal (3-Dimentional) filters [1-6] to remove the video noises. Spatial filters take only spatial information into account and as an effect can cause spatial blurring at high noise levels. This blurring effect can be reduced using both temporal and spatial information and the filtering performance can be improved at low noise levels also in this way.

A Wiener filter is utilized in the wavelet domain in order to remove image noise [2]. The author proposed fuzzy logic based image noise filter that takes directional deviations into account [3]. A recursive estimator structure has been proposed to estimate the clean image from the film-grain noisy image and the noise is considered to be related to exposure time in the form of non-Gaussian and multiplicative structure [4]. A pixel based spatio-temporal adaptive filter that calculates new pixel values adaptively using the weighted mean of pixels over motion compensated frames has been proposed in [5]. An edge preserving spatio-temporal video noise filter that combines 2D Wiener and Kalman filters has been presented in [6].

The proposed idea shows a non-linear video noise filter which calculates new pixel values using a 3D window [7]. In this method the pixels are arranged with respect to related pixel values in the form of a 3D window according to their difference and average the pixels in the window after weighting them with respect to their order of sorting. And which gives good results in case of no- or slow local motion, but deforms image regions in cases of abrupt local motion. In the case of local motion, the 3D filtering performance of the method is highly reduced. In order to increase the $3 \mathrm{D}$ filtering performance of the method proposed in [8] and Video denoising uses 2D and 3D dual-tree complex wavelet transforms. [9] Shows 2D wavelet based filtering and temporal mean filtering that uses pixel based motion detection.

The author of [10] proposed a wavelet transform based video filtering technique that uses spatial and temporal redundancy. In [11], a content adaptive video denoising filter has been proposed recently. This method filters both impulsive and non-impulsive noise but the filtering performance is highly reduced in case of Gaussian noise with high variance. In this work, a new pixel based spatio-temporal video noise filter that takes motion changes and spatial standard deviations into 
account is proposed. The main objective of this paper is compressing the noise in archive video to produce a high visual quality for archive videos. [12] Propose hidden markov tree modeling for wavelet coeffient and provide high CPSNR, good quality and removes color artifacts while preserving edges. In [13] spatio-temporal filtering is used for film restoration, which provides good PSNR and Visual results.

The proposed technique shows [14] efficient and accurate wavelet based noise estimation method for white Gaussian noise in video sequences. Instead of motion estimation step, the surfacelet transform provides motion selective subband decomposition for video signals and it is low redundancy [15]. In the proposal [16] explains spatio- temporal predictive search block matching which provide reasonable computational cost and subjective visual quality. [17] Uses effective improvement on the OSA method in both 2 and 3D. Super resolution algorithm is not dependent on explicit motion estimation [18], provides artifacts free and high quality images. [19] Is obtained based on an efficient quad tree decomposition of the learned dictionary and overlapping image patches.

The result shows the comparison between DWT and Dual Tree complex wavelet transforms [20]. Author developed greedy basis selection algorithm for ADDWP, which has lower computational complexity [21] as well as boundary extension method can be used to improve the quality of the first and last served frames. Bilateral filtering can be decomposed into a number of constant time spatial filters [22]. The main advantages are higher PSNR, Faster because of parallel implementation, and small memory footprint that is two percentages. Video sequences are considered as volume rather than a sequence of frames and not require any motion estimation [23]. Inpainting technique is flexible but time consuming which provides filling in the missing part of the video with the most appropriate data. Removing serious mixed noise from the video data and the mixed noises are Gaussian and impulse noise [24].

The low complexity method is [25] because reusing motion estimation resources from the video coding module for video denoising. [26] Provide data parallel, deeply multithreaded cores and high memory bandwidth. Video's can be denoised by the use of motion compensation that is without detecting the motion [27], which can be done through the correlation of neighboring frames that means selective block matching technique. In this paper white Gaussian noise is considered. So complexity is reduced but the quality of the image is normal. [28] Describes the motion estimation technique can be done through optical flow method and which is integrated with nonlocal means frame work with noise level estimation. In this paper, [29] present a new patch-based video denoising algorithm capable of removing serious mixed noise from the video data. By grouping similar patches in both spatial and temporal domain, formulate the problem of removing mixed noise as a low-rank matrix completion problem, which leads to a denoising scheme without strong assumptions on the statistical properties of noise. Many recent developed methods are present to solve the problem effectively. The robustness and effectiveness of our proposed denoising algorithm on removing mixed noise, e.g. heavy Gaussian noise mixed with impulsive noise.
The proposed idea of [31] uses spatiotemporal color filtering, nonlocal mean filtering for video denoising and for the color prediction the author uses inter frame and inter color prediction technique. [32] Shows 3D discrete cosine transform technique which can be used for real time video denoising. The author [33] gives detailed survey for video denoising and he used kalman filter and dynamic nonlocal mean algorithm for video denoising, which shows in real time the recursive algorithms are easy to realize. [34] Explains the video denoising can be done in openMP parallel processor to reduce the denoising time and the complexity. The noise considered is Gaussian white noise for denoising [35]. [36] Uses motion vector to detect the motion but the computational complexity is more. The author [37] says on about the combination of inter frame and inter prediction for color videos and shows the different color video denoising technique. The proposed technique [38] shows the quaternion vector filter for video denoising. But the author [39] says video denoising can be done efficiently through discrete complex wavelet transform. The [40-46] considered salt pepper impulse noise and 95\% quality received. In this paper [41-42] the author applied new technique as recursive video denoising using multi hypothesis. [43-45] Denoising is done through spatio temporal and DWT for Gaussian scale mixture. [44] Says color denoising can be done through demosaicking after the video denoising. [47] Shows the video denoising is applied to the digital TV.

The proposed technique gives new technique for video denoising that is poly view fusion like front, top and side views are considered for denoising using $2 \mathrm{D}$ approach. The various video denoising technique used are shown in the taxonomy of fig.1.Where MHMCF-multi hypothesis motion compensated spatial filter, NLSBP- nonlocal sparsity based priori, STGSM-spatio temporal Gaussian scale mixture, LPAICI-local polynomial approximation and intersection of confidence interval, and 3DSTP- 3d spatio temporal patches. The various motion detection techniques which used are shown in fig.2.Where STA3DKF-spatiotemporal adaptive 3d kalman filter, MCW - motion compensated wavelet filter, WF- wiener filter, GF - Gaussian filter, MHMCF - multi hypothesis motion compensated spatial filter, $\mathrm{BF}$-box filter, STLMMSEF - spatio temporal linear minimization mean square error filter, OSTA- optimal space time adaptation, SCBF - Self Cross Bilateral filter and AWAF- adaptive weighted averaging filter. Different noises which have been used are shown in fig.3. The ability to process a sequence in real time is dependent on three key factors: The speed of the algorithm, the frame rate required and the number of pixels. While considering all the above factors the real time video denoising can be done.

\section{MOTION ESTIMATION AND MOTION COMPENSATION}

By the use of recursive temporal filtering motion estimation enables us to fuse images and then reconstruct a high resolution image. Depend up on the size of the image; we have to decide the motion estimation technique. With a simple motion model very difficult to estimate motions. In this the simplest motion model is used, translational motion. But this motion technique will not be always valid. 


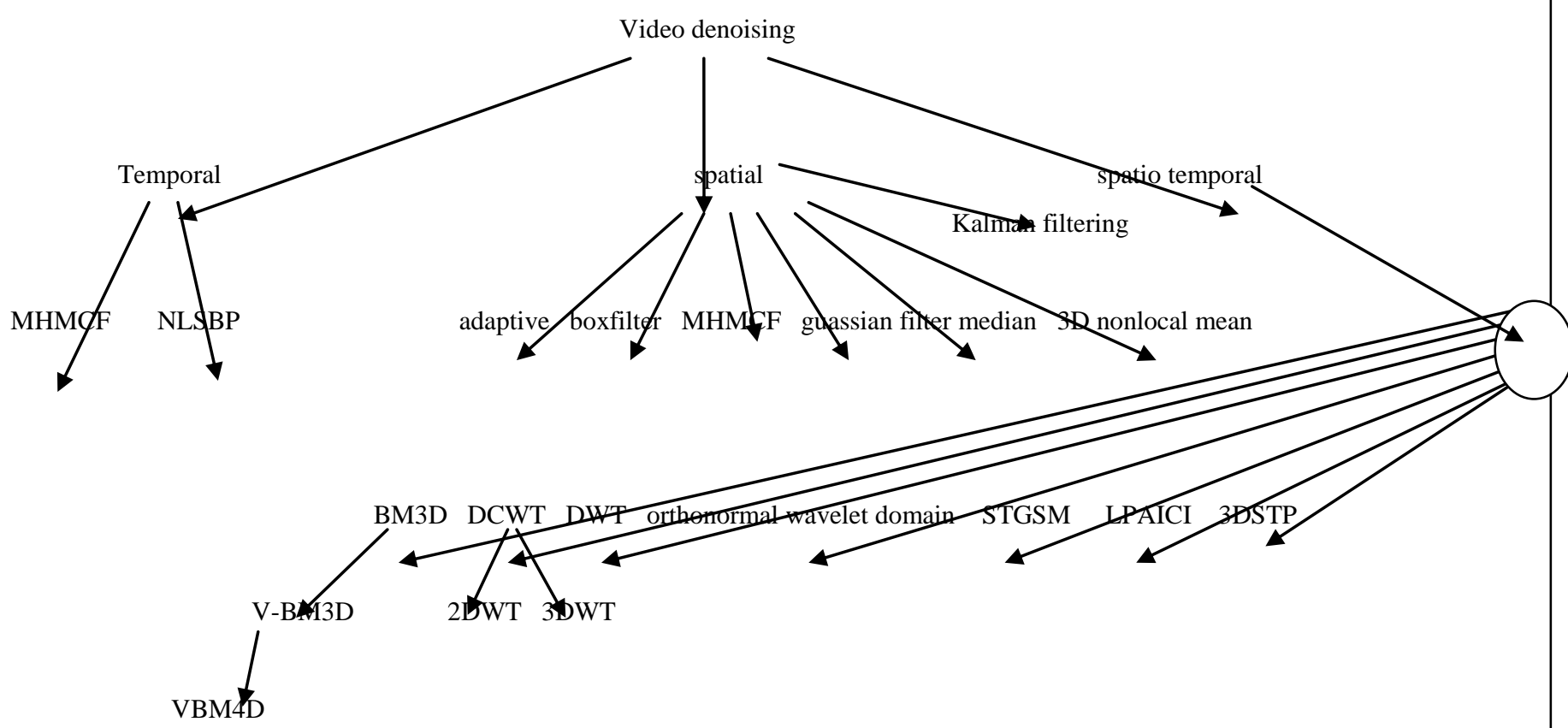

Fig.1 video denoising taxonomy

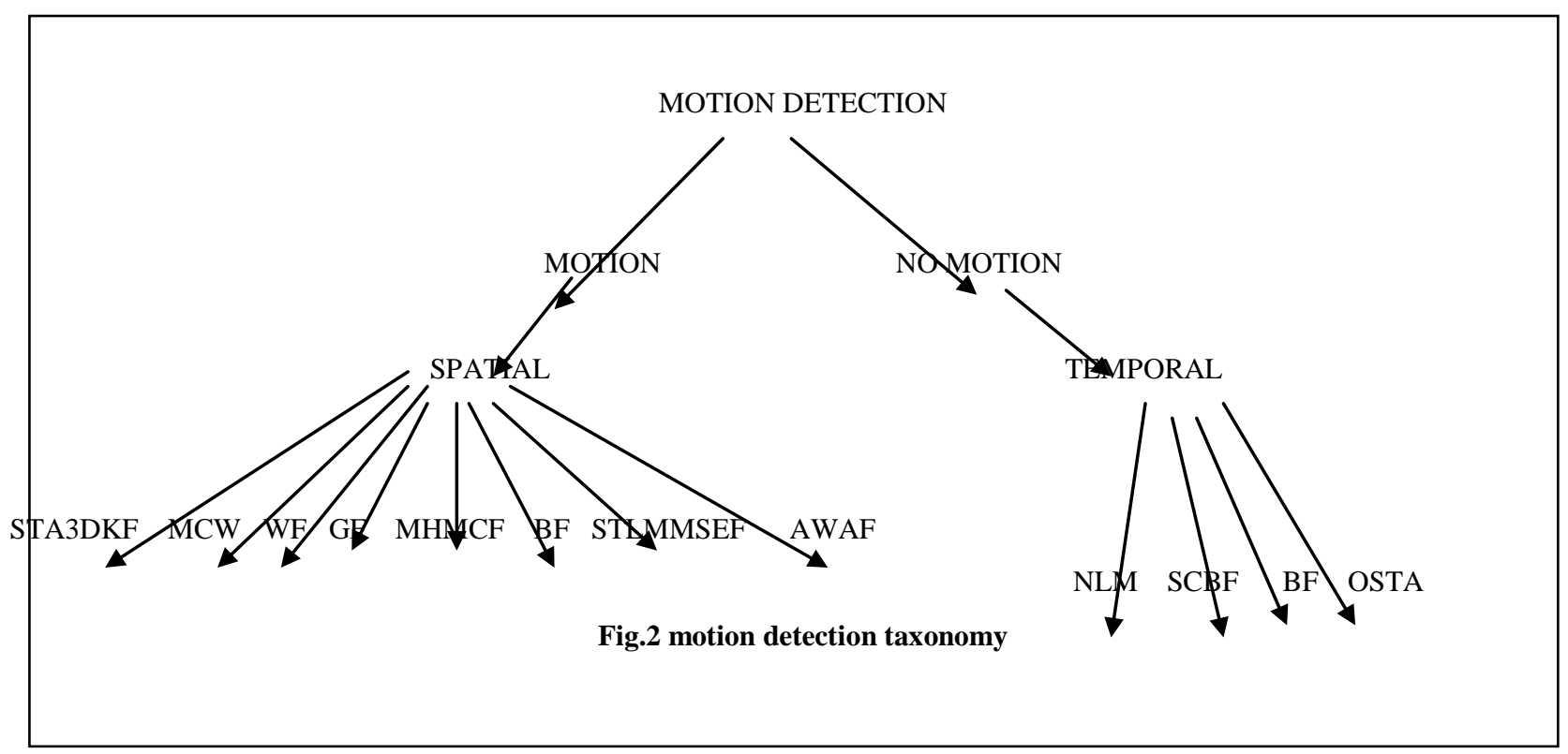




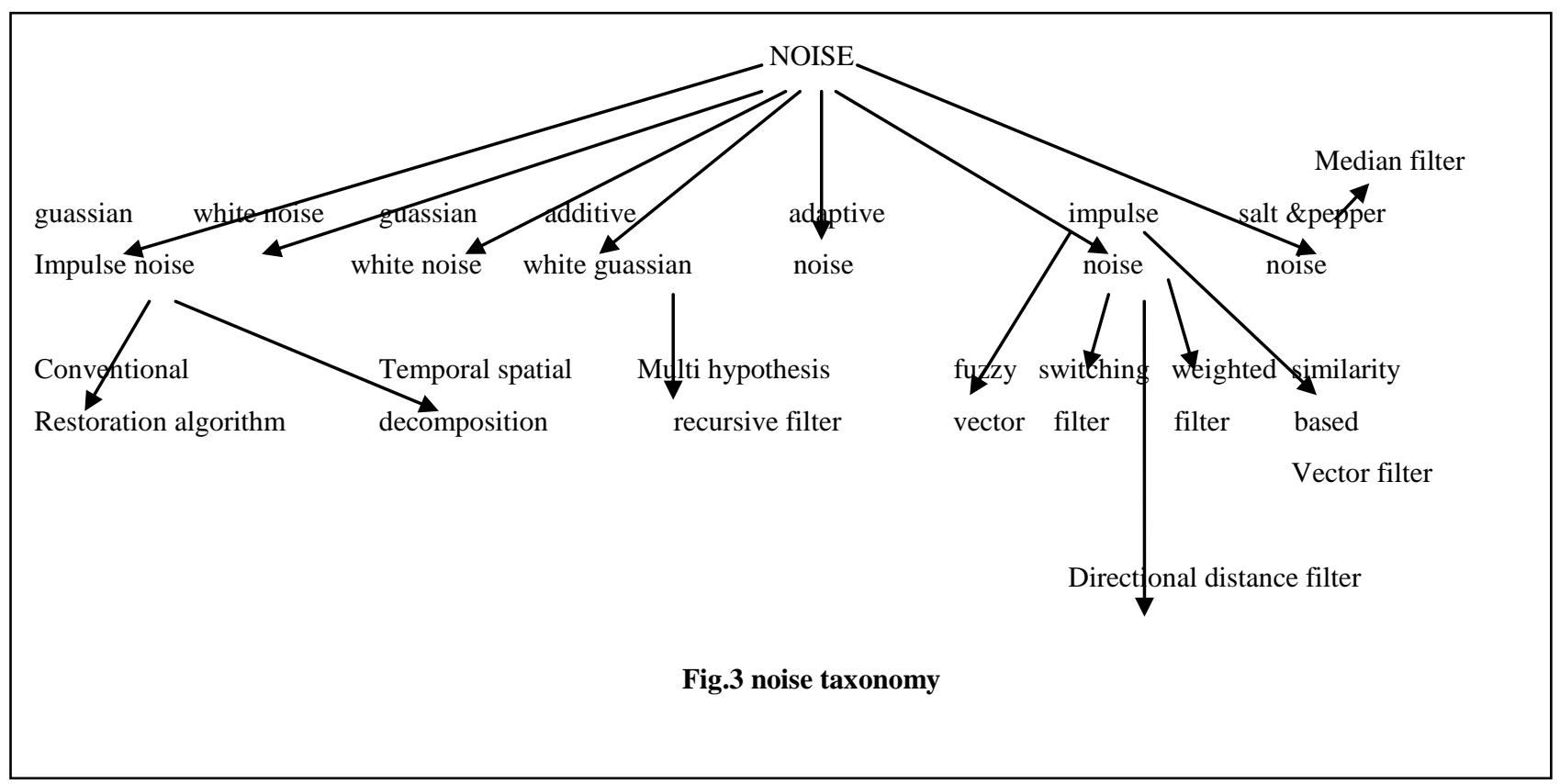

\subsection{Motion estimation}

Motion estimation is a partial redundancy between the neighboring frames. The selected frames are the reference frame and the subsequent frames are compared with the reference frame. The current frame contain current block and the reference frame contain the candidate block which is shown in fig.4. First step for motion estimation is to find the motion vector. Motion vector of each block is compared with the reference frame to get the motion compensation.

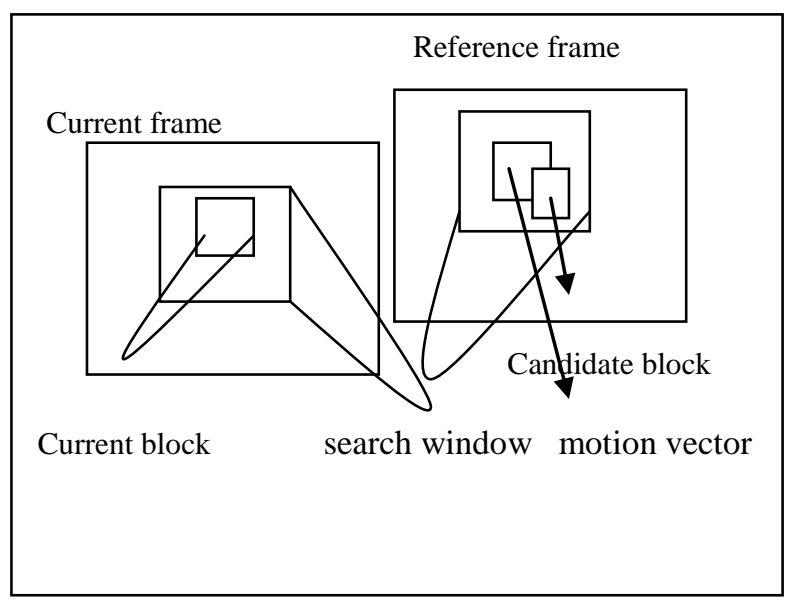

Fig.4 block matching

\subsection{Image Segmentation and compensation}

For large images estimation of motion is difficult. By the use of motion compensation for every frame two error frames are generated. They are combined and then segment the images into small pieces and estimate the motions for each piece to overcome this difficulty. Consider the below methods for segmentation.

\subsubsection{Fixed block base}

The simplest segmentation method is fixed block base. In this method, segment two frames (past and current frame/future frame) into a collection of fixed-size blocks in the same way, and then motion can be estimated between every pair of corresponding blocks of the past and current frames. Depend up on the block size motion will be estimated accurately but the local estimation becomes more difficult and unstable.

\subsubsection{Object or texture base}

Around the border areas of object or tetures have motion flow occlusions. This is the way to segment the images and then estimate the corresponding segments between the past and current/future frames.

\subsubsection{Motion base}

This is the most difficult method because the motion flow occlusions do not suitable for all the objects' borders. Motion estimation is difficult for larger segments. But the motion based segmentation method detects the occlusions and segment the images. High quality image is the result.

\subsection{Motion Models}

For the successful motion estimation motion models important. For unknown motion translational models are not flexible. To reduce the size, use some parametric fit. Due to small optical flow the estimation becomes unstable. Another way to proceed is to use affine or projective linear parametric motion models.

\section{EXPERIMENTAL RESULT}

The experimental result of salesman shows 3DSTP is performing very good for $\sigma=15$ and $\sigma=20$. The comparison table shows the details, which is in table 1 . 


\begin{tabular}{|c|c|c|}
\hline \multicolumn{3}{|c|}{ Salesman } \\
\hline Technique used & $\sigma 15$ & $\sigma 20$ \\
\hline MHMCF & 31.16 & 27.64 \\
\hline NLMC & 32.12 & 29.03 \\
\hline DNLM & 35.22 & 32.73 \\
\hline DCWT & 23.19 & 25.12 \\
\hline STA & 35.13 & 32.60 \\
\hline STGSM & 33.97 & 30.66 \\
\hline DWT & - & 33.25 \\
\hline 2DWT & - & 29.92 \\
\hline 3DWT & - & 29.35 \\
\hline WF & - & 37.508 \\
\hline MCW & 31.00 & 30.8 \\
\hline VBM3D & 40.44 & 36.07 \\
\hline VBM4D & 41.00 & 37.01 \\
\hline 3DSTP & 39.26 & 36.35 \\
\hline KNN & - & 32.5 \\
\hline $\begin{array}{c}\text { Orthogonal } \\
\text { wavelet domain }\end{array}$ & 31.71 & 30.16 \\
\hline LPAICI & 32.4 & 32.1 \\
\hline GF & 34.73 & 24.60 \\
\hline STLMMSEF & 26.49 & 21.04 \\
\hline OSTA & 33.89 & 33.66 \\
\hline AWAF & 33.78 & 32.51 \\
\hline
\end{tabular}

Table.1.comparison table of salesman for $\sigma 15$ and $\sigma 20$

\section{CONCLUSIONS}

Video signals are often contaminated during acquisition and transmission. Video denoising is the process of removing such distortions from a video signal [30]. The present techniques are very slow on processing and quality of Denoising is very poor for any real time applications like video conferencing and medical diagnosis. This research work is focused on proposing the methodologies for video denoising which is automatic, computationally lightweight and real time. In this paper, we have presented different methods and challenges of video denoising.

\section{REFERENCES}

[1] Malfait M, Roose D, Wavelet Based Image Denoising Using a Markov Random Field a Priori Model, IEEE Transaction on Image Processing, Vol. 6, No. 4, (1997) 549-565.

[2] Kazubek M,Wavelet Domain Image Denoising by Thresholding and Wiener Filtering, IEEE Signal Processing, Vol. 10, No. 11, (2003) 324-326.

[3] Van De Ville D, Van der Weken D, Nachtegael M, Kerre E. E., Philips W., and Lemahieu I, Noise Reduction by Fuzzy Image Filtering, IEEE Transaction on Fuzzy Systems, Vol. 11, No. 4, (2003) 429-436.
[4] Sadhar S. I., and Rajagopalan A. N, Image Estimation in Film-Grain Noise, IEEE Signal Processing Letters, Vol. 12, No. 3, (2005) 238-241.

[5] Ozkan M. K., Sezan I., and Tekalp A. M, Adaptive Motion Compensated Filtering of Noisy Image Sequences, IEEE Transaction on Circuits and Systems for Video Technology, Vol.3, No. 4, (1993) 277-290.

[6] Dugad R., and Ajuha N., "Video Denoising by Combining Kalman and Wiener Estimates", IEEE International Conference on Image Processing, Kobe, Japan, 4, (1999) 152-161.

[7] Zlokolica V., Philips W., Van De Ville D, A New NonLinear Filter for Video Processing, 3rd IEEE Benelux SPS-2002, Leuven, Belgium, (2002) 13-16.

[8] Selesnick I. W., and Li K. Y, "Video Denoising Using 2d and 3d Dual-Tree Complex Wavelet Transforms", In Wavelet Applications in Signal and Image Processing (SPIE 5207), San Diego, 5207, (2003) 607-618.

[9] Pizurica A, Zlokolica V, Philips W, Combined Wavelet Domain and Temporal Video Denoising, IEEE Conference on Advanced Video and Signal Based Surveillance (AVSS2003), (2003) page 334-341.

[10] Gupta N, Swamy M. N. S, and Plotkin E. I, LowComplexity Video Noise Reduction in Wavelet Domain, IEEE 6th Workshop on Multimedia Signal Processing, (2004) 239-242.

[11] Chan T.-W, Au O. C, Chong T. S, and Chau W.S,A Novel Content-Adaptive Video Denoising Filter, IEEE ICASSP, Philadelphia, PA, USA, Vol. 2, (2005) 649652.

[12] Nai-Xiang Lian, Vitali Zagorodnov and Yap Peng Tan, "Video denoising using vector estimation of wavelet coefficients", IEEE 2006, ISCAS2006.

[13] M Kermal Gullu, Oguzhan urhan and Sarp Eeturk, "Pixel domain spatio-temporal denoising for archive videos", ISCIS 2006, springer-Verlag Berlin Heidelberg 2006

[14] Vladimir Zlokolica, Aleksandra Pizurica, Wilfried Philips, Noise estimation for video processing based on saptio temporal gradients, IEEE signal processing letters,Vol.13, No.6, june 2006.

[15] Yue Lu And Minh N. Do, "video processing using 3D surfacelet transform", Urbana IL 61801,USA, 2006.

[16] Kostadin Dabov, Alessandro Foi and Karen Egiazarian, "video denoising by sparse 3D transform-domain collaborative filtering", EUSIPCO 2007, Poland, Sep. 3 7,2007 .

[17] Hae Jong Seo and Peyman Milanfar, "video denoising using higher order optimal space-time adaptation", ICASSP 2008,I EEE 2008.

[18] Matan Protter,Michael Elad, Hiroyuki Takeda, and Peyman Milanfar, "Generalizing the nonlocal- means to super resolution Reconstructio", IEEE transaction on image processing, Volume18, Number1,Jan 2009.

[19] JulienMairal, GuillermoSapiro, MichaelElad, Learning multiscale sparse representation for image and video restoration, IMA Preprint Series of 2168.

[20] Rasha Orhan Mahmoud and Mahmoud T. Faheem, Comparison between DWT and Dual Tree Complex 
wavelet transform in video sequences using wavelet domain, infos2008, march27-29, eario-Egypt 2008.

[21] Jingyu Yang, Yao Wang, Wenli Xu and Qionghai Dai, "Image and video denoising using adaptive dual tree Discrete wavelet packets", IEEE transaction on circuits and systems for video technology.

[22] QuingXiongYang, Kar-Itan-Tan and Naredra Ahuja, Real time O(1) Bilateral filtering, IEEE 2009.

[23] Mahmoud Ghoniem, Yoursef chahir and Ahdrrahim Elmoatar," Non local video denoising, simplification and inpainting using discrete regularization on graphs", 2009 ELSEVIER.

[24] HuiJi,ChaoqiangLiu, Zuoweishon and Yuhongxu,"Robust video denoising using lowranKmatrixcompletion,http.//media.xiph.org/video/de rf.

[25] Ljubomir Jovanov, Aleksandra Pizurica, Stefan schulta,Peter Schelkon,Adrian munteanu, Etiennakerre and wlfried Philips," Combined wavelet domain and motion compensated video denoiding based on video codec motion estimation method", IEEE , Vol.19,No.3, March 2009.

[26] Roberto Di Salvo, Carmelo Pino., Image and video processing on CUDA: state of the Art and Future Directions, ISBN-978-1-61804-046-6.

[27] Florian Luisier, Thierry Blue, Michael Unser, "surelet for orthonormal wavelet domain video denoising", 2010 IEEE ,Vol.20, No.6, JUNE 2010.

[28] William T. Freeman, C Liu, "A High quality video denoising algorithm based on reliable motion estimation”, 2010 Spinger, ECCV2010.

[29] Radha s.Shirbhat, Nitish D.Mishra and Rasika P.Pande, "video survilence system using motion detection A Survey", IJANA,Vol.3,I-5,P-(19-22),2012.

[30] G.Healey, R.Kondepudy, Radiometric ccd camera calibration and noise estimation, IEEE, PAMI, 267-276, 1994.

[31] Jingjng Dai, Oscar C Au, Chao Pang, feng Zou, Combined inter frame and inter color prediction for color video denoising, 2012 IEEE international conference on multimedia and Expo

[32] Michal Joachimiak, Dmytro Rusanovsky, Miska, Moncef, "Multiview 3D video denoising in sliding 3D DCT Domain”, EURASIP,Aug 27-31, 2012

[33] Yubing Han, Rushan Chen, "Efficient video denoising based on dynamic nonlocal means", Elsevier,Image and vision computing b30(2012) 78-85

[34] [34]B.Dolwithayakul, C. chantrapornchai, N. Chumchob, Real time parallel spatio temporal video denoising scheme on Multicore processor, IEEE 2012
[35] Xiaolin Tian, LichengJiao, Ying Duan, "Video denoising via spatially adaptive coefficient shrinkage and threshold adjustment in surfacelet transform domain", Springer,15 may 2012

[36] MatteoMaggioni, GeacomoBoracchi, AlessandroFoi, KarenEgiazarian, Video denoising, Deblocking and enhancement through separable 4D nonlocal spatio temporal transform, IEEE Transaction on image processing, Volume21, Number9, Sep 2012

[37] JingjngDai, OscarCAu, ChaoPang fengZou, Combined inter frame and inter color prediction for color video denoising, IEEE 2011

[38] Lianghai Jin, Hang Liu, Xiangyang, Enmin Sony, "Quarternian based impulse noise removal from color video sequences", IEEE,2011

[39] Hossein Rabbani, Video deblurring in complex wavelet domain using local laplaceprior for enhancement and anisotropic spatially adaptive denoising for PSF detection, IEEE 2010

[40] YiWan, QiqiangChen, A Novel quadratic type variational method for efficient salt and pepper noise removal, IEEE 2010

[41] JingjngDai, OscarCAu, ChaoPang, fengZou, XingWen, Color Video denoising based on adaptive color space conversion, IEEE 2010

[42] H.Tan,F.Tian, Y. Qiu, S.Wang, J.Zbang, "Multihypothesis recursive video denoising based on separation of motion state", IET image process,2010,vol.4,Iss.4,pp.261-268

[43] HijeshVarghese, ZhouWang, Video denoising based on spatio temporal guassian scale mixture model, IEEE Transaction on circuits and system for video technology, volume20, Number7, July2010

[44] LeiZhang, WeishengDong, Xiaolin Wu, GuangmingShi, Spatiotemporal color video reconstruction from noise CFA sequenc, IEEE Transaction on circuits and system for video technology, volume20, Number6, June2010

[45] ShgongYu, M.Omair, Ahmad, M.N.S.Swamy,Video denoising using motion compensated 3D wavelet transform with integrated recursive temporal filtering, On circuits and system for video technology, volume20, Number6, June2010

[46] K. Aisarya, v Jayaraj, D. Ebenezer, A New and efficient algorithm for the removal of high density salt and pepper noise in image and video, IEEE computer society, 2010

[47] Zhang, JangWooHan, JunHyungKin, sungJerko, A gradient salient based spatio temporal video noise reduction method for digital TV, IEEE.2010

[48] KaiZeng, Zhouwang, polyview fusion: a strategy to enhance video denoising algorithm, IEEE.2010 